DOI 10.22448/AMJ.2017.3.58-59

\title{
EFFECT OF CHINESE MEDICINE YIQI HUOXUE JIEDUMETHODONTHE EXPRESSIONOF INFLAMMATORY CYTO- KINES IN PATIENTS WITH SEVERE SEPSIS
}

\author{
QunLiang1Lixiang Xie2
}

\author{
1The First Affiliated Hospital of Heilongjiang University Of Chinese Medicine ; 2Heilongjiang University Of Chinese \\ Medicine
}

Address:No.26 Heping Road,Xiangfang District,Heilongjiang,Harbin,China. E-mail:liangqun1@sina.com

Abstract: $\quad$ Sepsis is a syndrome of life-threatening organ dysfunction caused by host reaction caused by loss of control. At present, the treatment of sepsis has made great progress, but the effect is poor, the mortality rate is still high. It is necessary to find a drug can effectively prevent or treat sepsis drugs have been urgent. Chinese medicine plays a role can not be ignored in the treatment of sepsis, the efficacy of patients after treatment is more significant. Chinese medicine preparations HuaXian capsules has the effect of Yiqi Huoxue Jiedu. Through the clinical experimental study shows that sepsis on the basis of routine treatment of Western medicine with HuaXian capsules in Yiqi Huoxue Jiedu method, can make the patient's inflammatory cytokine expression level was significantly reduced, with good clinical efficacy.

key words:Yiqi Huoxue Jiedu method;Sepsis; Inflammatory Cytokines;TNF-a、IL-6 and IL-10

Objective: To observe the effect of Chinese medicine Yiqi Huoxue Jiedu method on the expression level of TNFa. IL-6 and IL-10 in patients with severe sepsis.

Materials and methods: The patients in this study were provided by the Department of critical medicine of our hospital, 70 patients with sepsis from January 2015 to December 2016 were randomly divided into control group ( $n=36$ ) and treatment group $(n=34)$. All patients met the diagnostic criteria for sepsis developed by the 2012 edition of the "International Guidelines for Severe Septic and Septic Shock Management" and informed family and family informed consent.

All patients were treated in accordance with the principle of the 2012 edition of "International Guidelines for Severe Septic and Septic Shock Management": (1) the control group was given routine treatment of sepsis: (2) the treatment group was treated with Yiqi Huoxue Jiedu method Huaxian Capsule on the basis of routine western medicine, the Chinese medicine compound preparation composed of ginseng, Astragalus salvia, angelica, chuanxiong, Poria, Atractylodes, radix rehmanniae, licorice and other drugs, this prescription has the effect of Yiqi huoxue jiedu. Take the above traditional Chinese medicine, according to the Chinese Pharmacopoeia (2005 Edition) Chinese medicine capsule preparation method, HuaXian capsule is prepared by the First Affiliated Hospital of Heilongjiang University Of Chinese Medicine pharmaceutical factory, specifications for the $0.75 \mathrm{~g} / \mathrm{kg}$. The use of nasal feeding, the dose for adults $1.5 \mathrm{~g} /$ times, 3 times a day. Two groups were treated for 14 days. The levels of inflammatory cytokines TNF-a, IL-6 and IL-10 、 the hospitalization time and the mortality rate in 28days in the sepsis patients were compared between the two groups and the peripheral venous blood was collected at the 3rd, 5th and 7th day before treatment and after treatment. To investigate the effect of Yiqi Huoxue Jiedu method on the expression of inflammatory cytokines in patients with severe sepsis.

Results and discussion

There was no significant difference in the concentrations of TNF-a, IL-6 and IL-10 between the two groups before treatment ( $P>0.05$ ). The concentration of TNF-a, IL-6 and IL-10 in the t-wo groups of sepsis patients decreased after treatment, and the decrease rate of the treatment group was significantly higher than that of the control group $(P<0.05)$; The duration of ICU was significantly shorter in the treatment group than in the control group $(P<0.05)$;The APACHE II score of the treatment group was significantly lower than that of the control group $(P<0.05)$. The mortality rate at 28 days was lower than that of the control group.

Thisstudyshowsthatusing HuaxiancapsulewithYiqiHuoxueJiedumethodinthetreatmentofsepsispatientscan improveclinicalefficacy, compared with thetreatmentgroupand controlgroup, PatientsinthetreatmentgroupcanshortentheICUtime, reducetheAPACHE II score, thesepsispatientsTNF-a, IL-6, IL-10serumconcentrationswerereduced, reducingthemortality.

\section{References:}

1.M Shankar-Hari,GS Phillips,ML Levy,et al.Developing a New Definition and Assessing New Clinical Criteria for Septic Shock:For the Third International Consensus Definitions for Sepsis and Septic Shock (Sepsis-3)[J].Radiology,2016,227 (1):283-288.

2.N Semeraro,CT Ammollo, F Semeraro,et al.Sepsis, thrombosis and organ dysfunction[J]. Thrombosis Research, 2012, 129(3): 290-295.

3.Q Liang,H Liu,H Xing,Y Jiang,et al.Urinary UPLC-MS metabolomics dissecting the underlying mechanisms of Huaxian capsule protects against sepsis[J].RscAdvances, 2016,6(46):40436-40441.

4.Ying Wu,Zhaobo Cui, Hongshan Kang,et al.Effects of ulinastatin on serum tumor necrosis factor alpha, interleukin 10, troponin I and CRP levels in patients with sepsis[J]. Journal of Bengbu Medical College, 2016, 41(12):1635-1638.

5.Dong Zhang,Xingqun Yu.Research Progress on the treatment of sepsis with Jiedu Yiqi Huoxue method[J].Journal of Emergency in Traditional Chinese Medicine,2013,22(7):1191 
6.Q Liang,H Liu,H Xing,et al.High-resolution mass spectrometry for exploring metabolic signatures of sepsis-induced acute kidney injury[J].Rsc Advances,2016,6(36):29863-29868.

7.Q Liang, H Liu, T Zhang,et al.Metabolomics-based screening of salivary biomarkers for early diagnosis of Alzheimer's disease[J]. Rsc Advances,2015,5(116):96074-96079.

8.Guoye Ji,Bin Wang,Jiang Du,et al.Effects of hemoperfusion at different time on blood IL-6 and TNF-a levels in rabbits with sepsis[J]. Modern Medical Journal,2011,39(2):129-133.

\title{
DOI 10.22448/AMJ.2017.3.59-60
}

\section{FIVE 1 TECHNOLOGY FINDINGS IN THE PERMEATION RATE OF APPLICATION AT ACUPOINT}

\author{
Rui Wang1, Qun Feng, Yanhong Wang, Jing Yang and Yongji Li* \\ Heilongjiang University of Chinese Medicine, Harbin, Heilongjiang
}

1First author: A/Prof. Rui Wang, Heilongjiang University of Chinese Medicine, Harbin, Heilongjiang, 150040. Tel: 0451-87266893, E-mail:wrdx@sina.com

*Correspondence author: Prof. Yongji Li, Heilongjiang University of Chinese Medicine, Harbin, Heilongjiang, 150040. Tel: 0451-87266893; E-mail: liyongji2009@163.com

Abstract Objective The acupoint is an important role in the traditional Chinese medicine. However, it is unclear that the potential mechanism of acupoint effect, and whether had other characters. We conjecture that acupoint application can enhance the permeation rate of skin.

Methods This study, we through application fluorochrome at Zusanli (ST 36) and general site of rabbits and employ Fluorescence in vivo Endomicroscopy (FIVE 1) to obtain the images of connective tissue under the skin. And compare the fluorescence images of acupoint and general site in 5, 10 and $15 \mathrm{~min}$.

Results the acupoint group was more quickly to achieve the fluorescence intensity which could to observe the connective tissue.

Discussion It is revealed that application at Zusanli (ST 36) could improve the permeation rate of fluorochrome.

Key words: acupoint application, Zusanli (ST 36), FIVE 1

Acupoint is an important role in the traditional Chinese medicine, and it is the basis of manipulation and acupuncture. It has been employed to treat various diseases more than 2000 years in East Asia by inserting and manipulating in acupoints1. Acupuncture has become an important complementary and alternative medicine treatment in western countries2. However, the potential mechanism of acupoint has been difficult to illuminate. Thus, we conjectured that application at acupoint could change the permeation rate of skin.

Objective To assess the permeation rates of acupoint and general site employ the FIVE 1, and it will reveal the advantage of acupoint application.

Materials and methods At first, injected anesthetics in ear marginal vein to anesthetize. Random choice five rabbits as test group to point application fluorochrome at Zusanli (ST 36), and the others five rabbits as control group were application fluorochrome at the general site which was in the left $2 \mathrm{~cm}$ of ST 36. Then FIVE 1 probe was pierced at the subcutaneous tissue to observe the level of permeability of fluorochrome for 5, 10 and $15 \mathrm{~min}$.

Results and discussion After 5 min, the images of connective tissue were shown as Fig. 2. The fluorescence intensity of Zusanli (ST 36) group was enough to observe the characteristic of connective tissue, and the general site group was too dark to be able to observe the connective tissue.
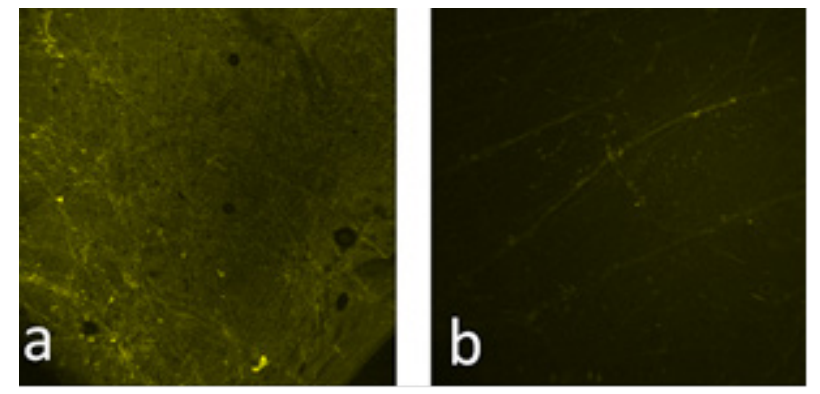

Fig. 2 The images of connective tissue. a was the Zusanli (ST 36) image; b was the general site group image.

As shown in Fig. 3. After 10 min, the connective tissue of two groups was all clear, but Zusanli (ST 36) group was more brightly than control group. 